\title{
Horticulture Evaluation and Genetic Identification of Selected Le-Conte Pear Clone
}

\author{
Hanaa M. Sherif, Samia A. Asaad and Nahla A. Abd-Elfatah \\ Horticulture Research Institute, Agriculture Research Centre, \\ Cairo, Egypt.
}

\begin{abstract}
A SELECTED clone of Le- Conte pear was evaluated in $A$ comparison to Le-Conte old cultivar. Flowering of the selected clone was earlier by 7-10 days. Maturity was 25-30 days earlier than the old cultivar. Whereas it produced fruits fewer than the old cultivar Le-Conte under the same conditions. No significant differences were observed between the new clone and the old cultivar within the vegetative growth . However, fruit physical prosperities increased significantly in the selected clone compared to the old cultivar. Chemically, the selected clone revealed a higher ratio of T.S.S / acidity than in Le-Conte cultivar. The same trend was noticed within total and reducing sugars. Yield was higher in the old cultivar than in the selected clone.

Screening of DNA by RAPD marker showed a total number of 74 amplicons with an average of 7-4 amplicon/ primer when ten primers were used. The highest number of polymorphic amplicon (2) produced by OPA17. The size of fragment varied from 280-1790 bp. Two negative unique markers were detected with the selected clone by OPA 17. These markers were located at 550 bp and150 bp. Meanwhile, OPG 06 revealed an unique positive marker at 450 bp.The estimated similarity between the selected clone and the old cultivar was 95.3 .
\end{abstract}

Pear is considered an economically important fruit among other deciduous fruit trees and the fourth among all fruits in its global distribution (Vanneste et al., 2002). In Egypt, productivity of "Le Conte", the main pear cultivar (resulted as a hybrid between Pyrus Communis x Pyrus Seratonia) significantly varies from one year and location to another (Sanaa et al., 2012).

Evaluation and selection of a new clone or strain play an important role for improving fruit quality especially under Egyptian condition, while in pear genotype spread in a narrow wide. Le-Conte pear cultivar is the only cultivated one in Egypt. European pear (Pyrus commuins L.) is grown in cold, moderate climate conditions. Commercial orchards consist of "Le-Conte" pear as the main cultivar, the yield varies from year to year. This variability has been attributed mainly to lack of adequate cross pollination; as well as, other factors can affect fruit set and yield like as fire blight ( Lee, 1948).

In Egypt, cultivated area reached 20400 feddans that produced about 124800 tons with an average production of 6.12 tons/ feddan according to Ministry of 
Agricultural (2010). Many researchers have been attempted to increase productivity and quality of fruits. Pear production ranged from 5-6 tones per feddan with a farm gate currently supplies only $30-40 \%$ of market requirements (Mohamed et al., 2012).

Plant varieties have certain characteristics including size, colour, flavor ...ect, but which have occurred naturally without the intervention of man (Spencer, 2007). Plant strain characteristics have been "fixed" by nature and can be propagated by seed with resulting plants coming true to the parent plants with only minor differences. A cultivar is a plant which has been bred for desirable characteristics such as size, colour, yield, disease resistance etc., by means of hybridization i.e. the crossing of two or more different varieties of plants (Bailey, 1923). Clone, in the case of fruit always done by vegetative means in the form of cuttings, division, grafts, and budding (Trehane, 2004).

RAPD markers were developed by Williams et al. (1990). RAPD technique utilizes single arbitrary 10-mer oligonucleotides as primers to amplify discrete fragments of DNA in low-stringency polymerase chain reaction (PCR). RAPD markers require no prior knowledge of the DNA sequence, which makes them very suitable for investigation of species that are not well known. The method is fast and easy to perform (Williams et al., 1993).

The objectives of the study were to evaluate a superior clone of local pear tree based on growth and yield performance, physical and chemical characteristics of the fruit and to develop RAPD marker based DNA fingerprinting for identification of the selected superior clone.

\section{Materials and Methods}

The present study was carried during two consecutive seasons from 2012/2013 on Le-Conte pear trees as well as selected pear clone budded on Pyrus betulaefolia. All trees were (3 replications, old cultivar and one tree, selected clone) 5 years -old, planted at $5 \times 5$ and grown in sandy soil under drip irrigation at El-Hag Ibrahim El-Mongy farm at Kaffer Dawood village, Sadat city, El-Mounifia Governorate, Egypt. Four branches of two years old in four directions were tagged to determine the main characteristics of the tree as follow:

Data of both flowering and vegetative growth were recorded during the growing seasons 2012-2013, including dates of both beginning of flowering and fruit set during the studied seasons.

\section{Vegetative growth}

At the end of the growing seasons (mid August), four shoots (2 years-old) in four direction of selected clone and old cultivar trees were labeled for measuring parameters ,

- Shoot length (cm).

- Shoot diameter (cm).

Egypt. J. Hort. Vol. 41, No. 1 (2014) 
- Leaf area $\left(\mathrm{cm}^{2}\right)$.

- Tree height (m).

- Tree diameter (m).

- Trunk diameter $(\mathrm{cm})$.

Flowering and fruiting

Fruit set (\%)

The tagged four branches being mentioned before were used to determine the fruit set percentage. It was calculated in relation to the total number of flowers as follow:

Fruit set $(\%)=$ No. of developing fruit set /Total No. of flowers $\times 100$

Spurs (\%): It was calculated in relation to the shoot length as follow:

Spurs $(\%)=$ No. of spurs /shoot length $\times 100$

\section{Leaf chemical content}

Leaf chemical contents were determined in mid-August of both experimental seasons. Samples of 30 leaves /tree were taken at random from the previously vegetative spurs on tagged shoots of each tree. leaf samples were washed with tap water, oven dried at $70{ }^{\circ} \mathrm{C}$ to a constant weight and grounded. The ground samples were digested with sulphoric acid and hydrogen peroxide according to Evenhuis and De Waard (1980) Total nitrogen and phosphorus were determined according to Evenhuis (1978) and Murphy and Riley (1962) and the colorimetric method for total carbohydrates (\%) as outlined by Dubois et al. (1956). Potassium was determined by a flame Photometer model E.E/L. (Jackson, 1967). $\mathrm{Fe}, \mathrm{Zn}$ and $\mathrm{Mn}$ were measured by Perkin-Elmer atomic absorption spectrophotometer model $2380 \mathrm{Al}$, according to Jackson and Ulrich (1959). Leaf chlorophyll reading was recorded using Minolta chlorophyll Meter SPAD502(Minolta camera .Co, LtD Japan) at the field. Average of ten readings was taken from the middle of leaves from canopy tree (Yadava,1986).

Fruit physical properties

At picking date twenty fruits from each tree under study were chosen to determine the following parameters:

- Average fruit weight (gm).

- Average fruit volume $\left(\mathrm{ml}^{3}\right)$

- Average fruit length $(\mathrm{cm})$.

- Average fruit diameter $(\mathrm{cm})$.

- Shape index (weight / volume ratio and length /diameter ratio).

- Fruit firmness was estimated as firmness (Ib / $\mathrm{Inch}^{2}$ ) by Magness and Taylor pressure tester which has a standard 5/16 of inch plunger and recorded as $\mathrm{Ib} /$ inch $^{2}$.

- Normal and aborted seed number / fruit . 
Fruit chemical properties

- Total soluble solids (T.S.S) was determined by a hand refractometer,

- Acidity of fruit juice was determined (as malic acid) by titration with 0.1 normal sodium hydroxide with phenolphthalein as an indicator, according to A.O.A.C (2005).

- Total sugars \% content were determined according to Malik and Singh (1980).

- Total indols.

- Total phenols were determined with Folin-Coicagteu reagent according to the method of (Slinkard and Singleton, 1977).

- Total carbohydrates as outlined by Dubois et al. (1956).

Randomly Amplified Polymorphic DNA (RAPD)

RAPD-PCR3 Reactions

A set of ten random 10-mer primers (Table13) were used in the detection of polymorphism between a mutated selected clone of le-Conte pear and the commercial leconte pear. These primers were synthesized on an ABI 392 DNA/RNA synthesizer (Applied Biosystems) at AGERI. RAPD-PCR was carried out according to the procedure given by Williams et al. (1990) with minor modifications. The amplification reaction was carried out in 25 il reaction volume containing $1 \mathrm{X}$ PCR buffer, $1.5 \mathrm{mM} \mathrm{MgCl}_{2}, 0.2 \mathrm{mM}$ dNTPs, 1 ìM primer, $1 \mathrm{U}$ Taq DNA polymerase and $25 \mathrm{ng}$ template DNA.

\section{Thermocyling Profile and Detection of the PCR Products}

PCR amplification was performed in a Perkin-Elmer/GeneAmp® PCR System 9700 (PE Applied Biosystems) programmed to fulfill 40 cycles after an initial denaturation cycle for $5 \mathrm{~min}$ at $94^{\circ} \mathrm{C}$. Each cycle consisted of a denaturation step at $94^{\circ} \mathrm{C}$ for $1 \mathrm{~min}$, an annealing step at $36^{\circ} \mathrm{C}$ for $1 \mathrm{~min}$, and an elongation step at $72^{\circ} \mathrm{C}$ for $1.5 \mathrm{~min}$. The primer extension segment was extended to $7 \mathrm{~min}$ at $72^{\circ} \mathrm{C}$ in the final cycle.

The amplification products were resolved by electrophoresis in a $1.5 \%$ agarose gel containing ethidium bromide $(0.5 \mathrm{ug} / \mathrm{ml})$ in $1 \mathrm{X}$ TBE buffer at 95 volts. PCR products were visualized on UV light and photographed using a Polaroid camera. Amplified products were visually examined and the presence or absence of each size class was scored as 1 or 0 , respectively.

Data were statistically analyzed in random design according to the method of Sendecor and Cochran (1990), L.S.D at 5\% level was used for comparison between means of each treatment.

\section{Results and Discussions}

\section{Flowering development}

Table 1 shows dates of beginning flowering, fruit set and harvest during $2012 \& 2013$ season under study. The new clone was earlier in flowering, fruit set and picking dates compared with the old cultivar Le-Conte pear trees. Beginning of flowering was in 28/2and 27/2 compare with Le-Conte it was in $7 / 3$ in both

Egypt. J. Hort. Vol. 41, No. 1 (2014) 
seasons under study. Whereas, beginning of fruit set was in $2 / 3$ for the new clones and in 10/3 for the old cultivar (Le-Conte).Herein ,the suggested mature day for the new clo ne were in $22 / 6 \& 28 / 6$ in two seasons under study but the nature dates of Le-Conte trees was in late of July or early August. These results are in a same line with Chan -Chung et al. (1997), Bahlool et al. (2000) and Ito et al. (2004) in their studies on pears.

TABLE 1. Beginning of flowering, fruit set and fruit harvest during the two seasons 2012 / 2013.

\begin{tabular}{|l|c|c|c|c|c|c|}
\hline \multirow{2}{*}{ Genotype } & \multicolumn{2}{|c|}{ Beginning of flowering } & \multicolumn{2}{c|}{ Beginning of fruit set } & \multicolumn{2}{c|}{ Beginning of harvest } \\
\cline { 2 - 7 } & $\begin{array}{c}\text { Season } \\
\mathbf{2 0 1 2}\end{array}$ & $\begin{array}{c}\text { Season } \\
\mathbf{2 0 1 3}\end{array}$ & $\begin{array}{c}\text { Season } \\
\mathbf{2 0 1 2}\end{array}$ & $\begin{array}{c}\text { Season } \\
\mathbf{2 0 1 3}\end{array}$ & $\begin{array}{c}\text { Season } \\
\mathbf{2 0 1 2}\end{array}$ & $\begin{array}{c}\text { Season } \\
\mathbf{2 0 1 3}\end{array}$ \\
\hline $\begin{array}{l}\text { Selected } \\
\text { clone }\end{array}$ & $28 / 2$ & $27 / 2$ & $2 / 3$ & $2 / 3$ & $22 / 6$ & $28 / 6$ \\
\hline $\begin{array}{l}\text { Old } \\
\text { cultivar }\end{array}$ & $7 / 3$ & $7 / 3$ & $10 / 3$ & $10 / 3$ & $30 / 7$ & $1 / 8$ \\
\hline
\end{tabular}

Vegetative growth

Table 2 pointed to shoot length $(\mathrm{cm})$, shoot diameter $(\mathrm{cm})$, leaf area $\left(\mathrm{cm}^{2}\right)$ and chlorophyll content during seasons 2012 \& 2013.It was noticed that there are slight differences between the selected clone $(78.0 \& 72.02 \mathrm{~cm}$ and $1.00 \& 0.97$ $\mathrm{cm})$ and old cultivar trees $(76.83 \& 68.50 \mathrm{~cm}$ and $1.00 \& 0.90 \mathrm{~cm})$ in both shoot diameter and shoot length, respectively. The difference did not significant. While, leaf area was increased significantly in the selected clone $\left(32.20 \& 31.72 \mathrm{~cm}^{2}\right)$ compared with old cultivar $\left(30.35 \& 31.05 \mathrm{~cm}^{2}\right)$, respectively during the two seasons under study. Also, chlorophyll content was increased in the selected clone (57.62 \& 53.45) compare by the old cultivar (44.86 \& 50.09) in both Seasons. Those results are in agreement with Bahlool et al. (2000) and, Colaric et al. (2007) who mentioned that more nodes and leaves per shoots were formed as result of shoot growth reduction.

TABLE 2 Vegetative growth during the two seasons 2012 / 2013.

\begin{tabular}{|l|c|c|c|c|c|c|c|c|}
\hline \multirow{2}{*}{ Genotype } & \multicolumn{2}{|c|}{ Shoot length $(\mathbf{c m})$} & \multicolumn{2}{|c|}{$\begin{array}{c}\text { Shoot diameter } \\
(\mathbf{c m})\end{array}$} & \multicolumn{2}{c|}{ Leaf area $\left.\mathbf{( c m}^{\mathbf{2}}\right)$} & \multicolumn{2}{|c|}{$\begin{array}{c}\text { Chlorophyll } \\
\text { content }\end{array}$} \\
\cline { 2 - 9 } & $\begin{array}{c}\text { Season } \\
\mathbf{2 0 1 2}\end{array}$ & $\begin{array}{c}\text { Season } \\
\mathbf{2 0 1 3}\end{array}$ & $\begin{array}{c}\text { Season } \\
\mathbf{2 0 1 2}\end{array}$ & $\begin{array}{c}\text { Season } \\
\mathbf{2 0 1 3}\end{array}$ & $\begin{array}{c}\text { Season } \\
\mathbf{2 0 1 2}\end{array}$ & $\begin{array}{c}\text { Season } \\
\mathbf{2 0 1 3}\end{array}$ & $\begin{array}{c}\text { Season } \\
\mathbf{2 0 1 2}\end{array}$ & $\begin{array}{c}\text { Season } \\
\mathbf{2 0 1 3}\end{array}$ \\
\hline $\begin{array}{l}\text { Selected } \\
\text { clone }\end{array}$ & $78.0 \mathrm{~A}$ & $72.02 \mathrm{~A}$ & $1.00 \mathrm{~A}$ & $0.97 \mathrm{~A}$ & $32.20 \mathrm{~A}$ & $31.72 \mathrm{~A}$ & $57.62 \mathrm{~A}$ & $53.45 \mathrm{~A}$ \\
\hline $\begin{array}{l}\text { Old } \\
\text { cultivar }\end{array}$ & $76.83 \mathrm{~A}$ & $68.50 \mathrm{~A}$ & $1.00 \mathrm{~A}$ & $0.90 \mathrm{~A}$ & $30.35 \mathrm{~B}$ & $31.05 \mathrm{~A}$ & $44.86 \mathrm{~B}$ & $50.09 \mathrm{~B}$ \\
\hline
\end{tabular}

Means within each column followed by the same letter(s) are not significantly different at $5 \%$ level

\section{Leaf mineral content}

Data presented in Table 3 show the means value of leaf mineral content on both selected clone and old cultivar in both seasons under study (2012 \& 2013) . It is noticed that there were a differences between the selected clone and old cultivar in macro and micro -elements content. Either $\mathrm{N}(\%), \mathrm{K}(\%)$ or $\mathrm{Cu}$ 
$(\mathrm{mg} / \mathrm{kg})$ were increased in selected clone but P (\%), Fe (mg/kg), Mn (mg/kg) and $\mathrm{Zn}(\mathrm{mg} / \mathrm{kg})$ were higher in the old cultivar leaves.

TABLE 3. Leaf chemical content

\begin{tabular}{|l|c|c|c|c|c|c|c|}
\hline Genotype & $\mathbf{N} \%$ & $\mathbf{P \%}$ & $\mathbf{K \%}$ & $\begin{array}{l}\mathbf{C u} \\
\mathbf{m g} / \mathbf{K g}\end{array}$ & $\begin{array}{l}\mathbf{F e} \\
\mathbf{m g} / \mathbf{K g}\end{array}$ & $\begin{array}{l}\mathbf{M n} \\
\mathbf{m g} / \mathbf{K g}\end{array}$ & $\begin{array}{l}\mathbf{Z n} \\
\mathbf{m g} / \mathbf{K g}\end{array}$ \\
\hline $\begin{array}{l}\text { Selected } \\
\text { clone }\end{array}$ & 1.91 & 0.10 & 2.17 & 3.00 & 311 & 8.78 & 30.0 \\
\hline Old cultivar & 1.36 & 0.11 & 1.62 & 2.40 & 441 & 9.51 & 43.90 \\
\hline
\end{tabular}

Development of leaf area

Data in Table 4 and Fig .1 showed the development on leaf area from 22/6 until 1/8 in both seasons under study (2012 \& 2013). The selected clone recorded the highest value of leaf area than in the local cultivar $(22 / 6 \& 1 / 8)$ in both studied seasons.

TABLE 4. Development of leaf area

\begin{tabular}{|l|c|c|c|c|}
\hline \multirow{2}{*}{ Genotype } & \multicolumn{2}{c|}{$22 / 6$} & \multicolumn{2}{c|}{$15 / 8$} \\
\cline { 2 - 5 } & Season1 & Season2 & Season1 & Season2 \\
\hline Selected clone & $31.53 \mathrm{~A}$ & $32.93 \mathrm{~A}$ & $32.20 \mathrm{~A}$ & $31.72 \mathrm{~A}$ \\
\hline Old cultivar & $23.37 \mathrm{~B}$ & $25.67 \mathrm{~B}$ & $30.35 \mathrm{~B}$ & $31.05 \mathrm{~A}$ \\
\hline
\end{tabular}

Means within each column followed by the same letter(s) are not significantly different at $5 \%$ level

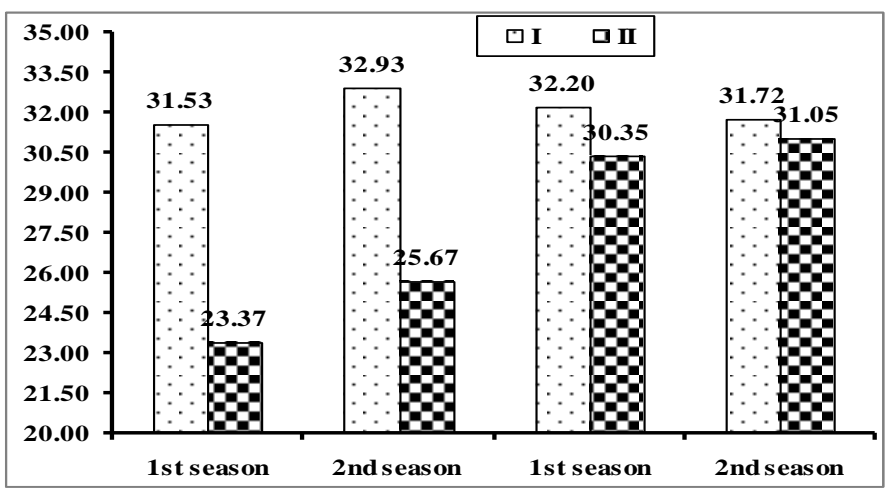

I. Selected clone II. 2-old cultivar Le-Conte

Fig. 1. Development of leaf area $\left(\mathrm{cm}^{2}\right)$

Tree shape

Regarding the tree height, tree diameter, trunk diameter and tree colour there were differences between the selected clone tree and old Le-Conte cultivar. It was noticed that the selected clone was gray in colour, medium in size, had trunk diameter of $41.5 \mathrm{~cm}$, tree diameter of $6.0 \mathrm{~m}$ and $3.5 \mathrm{~m}$ height. Whereas, old Le-Conte cultivar tree was brown, medium with trunk diameter of $35.0 \mathrm{~cm}$, tree diameter of $6.5 \mathrm{~m}$ and $3.75 \mathrm{~m}$ height (as being cleared in Table 5).

Egypt. J. Hort. Vol. 41, No. 1 (2014) 
TABLE 5. Tree character

\begin{tabular}{|l|c|c|c|c|c|}
\hline \multicolumn{1}{|c|}{ Genotype } & $\begin{array}{c}\text { Tree height } \\
(\mathbf{m})\end{array}$ & $\begin{array}{c}\text { Tree } \\
\text { diameter } \\
(\mathbf{m})\end{array}$ & $\begin{array}{c}\text { Tree } \\
\text { colour }\end{array}$ & $\begin{array}{c}\text { Diameter } \\
\text { of trunk } \\
(\mathbf{c m})\end{array}$ & $\begin{array}{c}\text { Tree } \\
\text { size }\end{array}$ \\
\hline Selected clone & 3.5 & 6.0 & Gray & 41.5 & Medium \\
\hline Old cultivar & 3.75 & 6.5 & Brown & 35.5 & Medium \\
\hline
\end{tabular}

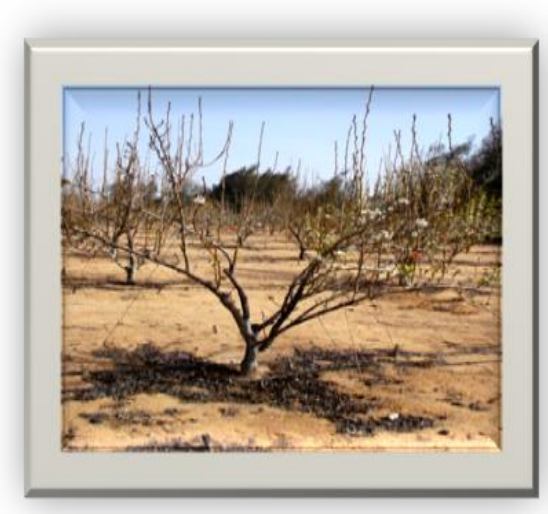

Fig. 2. Selected clone during dormant period

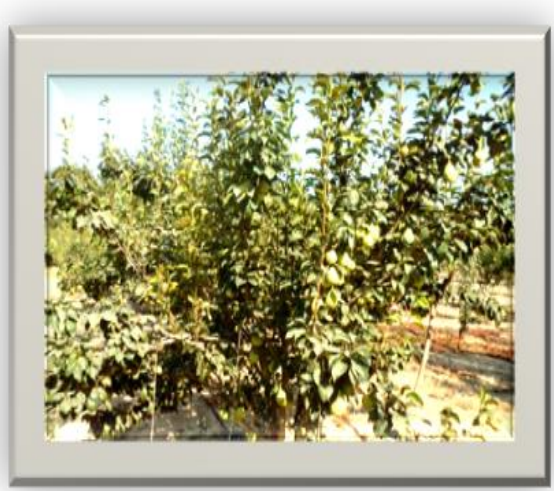

Fig. 3. Selected clone during growth season

\section{Tree fruiting}

As being cleared in Table 6 the selected clone gave a higher spurs number (15.33 \& 17.33) compared with the old Le-Conte cultivar trees (13.00 \&12.00) which subsequently affected fruit set.Whereas, old le-Conte cultivar trees gave a higher yield $58.76 \& 53.50 \mathrm{Kg}$ tree compared with the selected clone $52.41 \&$ 52.15 , respectively during seasons $2012 \& 2013$ under study. These findings are in harmony with Chan -Chung et al. (1997), Bahlool et al. (2000), Ito et al. (2004) and Fayek et al. (2011) in their studies on pears.

TABLE 6. Fruit set (\%) and yield (Kg/tree) during 2012/2013 seasons.

\begin{tabular}{|l|c|c|c|c|c|c|}
\hline \multirow{2}{*}{ Genotype } & \multicolumn{2}{|c|}{ Spurs \% } & \multicolumn{2}{c|}{ Fruit set (\%) } & \multicolumn{2}{c|}{ Yield(Kg/tree) } \\
\cline { 2 - 7 } & $\begin{array}{c}\text { Season } \\
\mathbf{2 0 1 2}\end{array}$ & $\begin{array}{c}\text { Season } \\
\mathbf{2 0 1 3}\end{array}$ & $\begin{array}{c}\text { Season } \\
\mathbf{2 0 1 2}\end{array}$ & $\begin{array}{c}\text { Season } \\
\mathbf{2 0 1 3}\end{array}$ & $\begin{array}{c}\text { Season } \\
\mathbf{2 0 1 2}\end{array}$ & $\begin{array}{c}\text { Season } \\
\mathbf{2 0 1 3}\end{array}$ \\
\hline $\begin{array}{l}\text { Selected } \\
\text { clone }\end{array}$ & $19.65 \mathrm{~A}$ & $24.06 \mathrm{~A}$ & $2.17 \mathrm{~A}$ & $2.14 \mathrm{~A}$ & $52.41 \mathrm{~B}$ & $52.15 \mathrm{~B}$ \\
\hline $\begin{array}{l}\text { Old } \\
\text { cultivar }\end{array}$ & $16.92 \mathrm{~B}$ & $17.52 \mathrm{~B}$ & $1.38 \mathrm{~B}$ & $1.60 \mathrm{~B}$ & $58.76 \mathrm{~A}$ & $53.50 \mathrm{~A}$ \\
\hline
\end{tabular}

Means within each column followed by the same letter(s) are not significantly different at $5 \%$ level 
Fruit physical properties

Obtained data during both 2012 and 2013 seasons cleared the differences between the selected clone and old Le-Conte cultivar fruit physical properties as being tabulated in Table 7. It is clear that all fruit physical properties were increased significantly in the selected clone compared with the old cultivar. The selected clone revealed the high values of fruit weight $(398.2 \& 318.8 \mathrm{~g})$, fruit size $\left(363.4 \& 317.20 \mathrm{~cm}^{3}\right)$, fruit length $(10.50 \& 10.73 \mathrm{~cm})$ and fruit diameter (8.71 \& 9.09). While the old Le-Conte cultivar detected the low values of fruit weight $(182.7 \& 186.2 \mathrm{~g})$, fruit size $\left(181.3 \& 178.9 \mathrm{~cm}^{3}\right)$, fruit length $(8.57 \&$ $8.69 \mathrm{~cm})$ and fruit diameter $(6.83 \& \mathrm{n} 6.69)$, respectively. These results are in harmony with Li-Tain et al. (1996) and Pierre (2001) on apple and Bahlool et al. (2000) on pear.

TABLE 7. Fruit physical properties during 2012 / 2013 seasons.

\begin{tabular}{|l|c|c|c|c|c|c|c|c|c|c|}
\hline \multirow{2}{*}{ Genotype } & \multicolumn{2}{|c|}{$\begin{array}{c}\text { Fruit weight } \\
(\mathbf{g})\end{array}$} & \multicolumn{2}{c|}{$\begin{array}{c}\text { Fruit size } \\
\left(\mathbf{c m}^{\mathbf{3}}\right)\end{array}$} & \multicolumn{2}{c|}{$\begin{array}{c}\text { fruit length } \\
(\mathbf{c m})\end{array}$} & \multicolumn{2}{c|}{$\begin{array}{c}\text { Fruit diameter } \\
(\mathbf{c m})\end{array}$} & \multicolumn{2}{c|}{$\begin{array}{c}\text { Fruit firmness } \\
\left(\mathbf{L b} / \mathbf{I n c h}^{\mathbf{2}}\right)\end{array}$} \\
\cline { 2 - 11 } & $\begin{array}{c}\text { Season } \\
\mathbf{2 0 1 2}\end{array}$ & $\begin{array}{c}\text { Season } \\
\mathbf{2 0 1 3}\end{array}$ & $\begin{array}{c}\text { Season } \\
\mathbf{2 0 1 2}\end{array}$ & $\begin{array}{c}\text { Season } \\
\mathbf{2 0 1 3}\end{array}$ & $\begin{array}{c}\text { Season } \\
\mathbf{2 0 1 2}\end{array}$ & $\begin{array}{c}\text { Season } \\
\mathbf{2 0 1 3}\end{array}$ & $\begin{array}{c}\text { Season } \\
\mathbf{2 0 1 2}\end{array}$ & $\begin{array}{c}\text { Season } \\
\mathbf{2 0 1 3}\end{array}$ & $\begin{array}{c}\text { Season } \\
\mathbf{2 0 1 2}\end{array}$ & $\begin{array}{c}\text { Season } \\
\mathbf{2 0 1 3}\end{array}$ \\
\hline $\begin{array}{l}\text { Selecte } \\
\text { d clone }\end{array}$ & $398.2 \mathrm{~A}$ & $318.8 \mathrm{~A}$ & $363.4 \mathrm{~A}$ & $317.2 \mathrm{~V}$ & $10.50 \mathrm{~A}$ & $10.73 \mathrm{~A}$ & $8.71 \mathrm{~A}$ & $9.09 \mathrm{~A}$ & $13.50 \mathrm{~A}$ & $13.55 \mathrm{~A}$ \\
\hline $\begin{array}{l}\text { Old } \\
\text { cultivar }\end{array}$ & $182.7 \mathrm{~B}$ & $186.2 \mathrm{~B}$ & $181.3 \mathrm{~B}$ & $178.9 \mathrm{~B}$ & $8.57 \mathrm{~B}$ & $8.69 \mathrm{~B}$ & $6.83 \mathrm{~B}$ & $6.69 \mathrm{~B}$ & $13.95 \mathrm{~B}$ & $13.90 \mathrm{~A}$ \\
\hline L.S.D & 11.51 & 7.157 & 14.02 & 6.680 & 0.699 & 1.348 & 1.301 & 1.595 & 0.762 & 1.452 \\
\hline
\end{tabular}

Means within each column followed by the same letter(s) are not significantly different at $5 \%$ level

Fruit shape index

Data being illustrated in Table 8 indicated that there were no significant differences in weight to volume (W/V). While length to diameter (L/D) recorded slight significant differences between the selected clone and the old cultivar.

TABLE 8. Fruit shape index during seasons 2012/2013.

\begin{tabular}{|l|c|c|c|c|}
\hline \multirow{2}{*}{ Genotype } & \multicolumn{2}{|c|}{ W/V ratio } & \multicolumn{2}{c|}{ L/d ratio } \\
\cline { 2 - 5 } & Season2012 & Season2013 & Season2012 & Season2013 \\
\hline $\begin{array}{l}\text { Selected } \\
\text { clone }\end{array}$ & $1.100 \mathrm{~A}$ & $1.04 \mathrm{~A}$ & $1.12 \mathrm{~A}$ & $1.140 \mathrm{~B}$ \\
\hline Old cultivar & $1.00 \mathrm{~A}$ & $1.04 \mathrm{~A}$ & $1.27 \mathrm{~A}$ & $1.30 \mathrm{~A}$ \\
\hline
\end{tabular}

Means within each column followed by the same letter(s) are not significantly different at $5 \%$ level

A significant differences were observed between the selected clone and old cultivar, in respect of normal seeds per fruit. The selected clone showed a higher number of normal seeds $(5.33 \& 5.33)$ than the old cultivar $(2.00 \& 1.67)$ in both studied seasons, respectively (Table 9). On the other hand, aborted seeds per fruit were detected in both of the selected clone and the old cultivar. Number of aborted seeds per fruit were lower in the selected clone than in the old cultivar (4.67\&4.67), (200\&2.00), respectively in both of the studied seasons.

Egypt. J. Hort. Vol. 41, No. 1 (2014) 


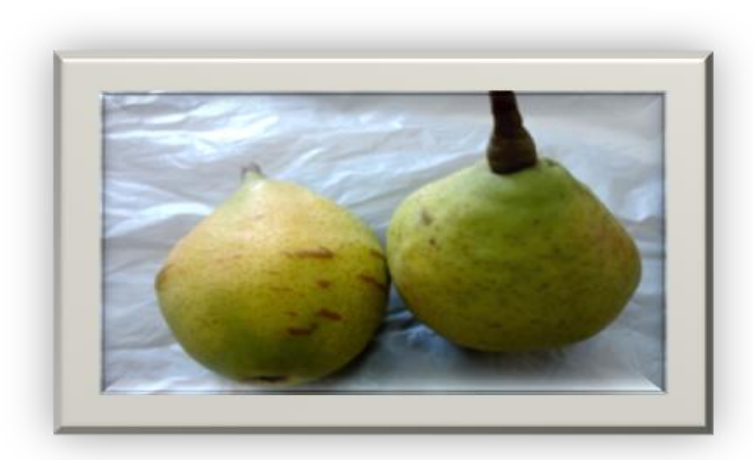

Fig. 4. Shap index of selected clone fruit

TABLE 9. Normal and aborted seed number/fruit during the two seasons (2012 \& 2013)

\begin{tabular}{|l|c|c|c|c|}
\hline \multirow{2}{*}{ Genotype } & \multicolumn{2}{|c|}{ Normal seeds } & \multicolumn{2}{c|}{ Aborted seeds } \\
\cline { 2 - 5 } & Season 2012 & Season 2013 & Season 2012 & Season 2013 \\
\hline Selected clone & $5.33 \mathrm{~A}$ & $5.33 \mathrm{~A}$ & $2.00 \mathrm{~B}$ & $2.00 \mathrm{~B}$ \\
\hline Old cultivar & $2.00 \mathrm{~B}$ & $1.67 \mathrm{~B}$ & $4.67 \mathrm{~A}$ & $4.67 \mathrm{~A}$ \\
\hline
\end{tabular}

Means within each column followed by the same letter(s) are not significantly different at $5 \%$ level

\section{Fruit development}

Fruit length development was significantly varied among the selected clone of Le-Conte pear and the old cultivar (Table 10 \& Fig.5). It is cleared that the selected clone had a higher length beginning from 16/6 to 22/7 compared with the old cultivar. Also, fruit diameter goes in the same trend of fruit length within the same dates (Table $10 \&$ Fig.6).

TABLE 10. Development of fruit length and fruit diameter during season 2013.

\begin{tabular}{|c|c|c|c|c|c|c|}
\hline \multirow{2}{*}{ Date } & \multicolumn{4}{|c|}{ Mean length (cm) } & \multicolumn{2}{c|}{ Mean diameter (cm) } \\
\cline { 2 - 7 } & Selected clone & Old cultivar & Mean & $\begin{array}{c}\text { Selected } \\
\text { clone }\end{array}$ & Old cultivar & Mean \\
\hline $16 / 6$ & $8.20 \mathrm{~b}$ & $5.43 \mathrm{~d}$ & 6.82 & $6.78 \mathrm{~b}$ & $3.93 \mathrm{f}$ & 5.36 \\
\hline $22 / 6$ & $10.13 \mathrm{a}$ & $6.57 \mathrm{c}$ & 8.35 & $9.17 \mathrm{a}$ & $5.20 \mathrm{~cd}$ & 7.19 \\
\hline $1 / 7$ & $7.93 \mathrm{~b}$ & $5.63 \mathrm{~cd}$ & 6.78 & $6.17 \mathrm{bcd}$ & $4.30 \mathrm{ef}$ & 5.24 \\
\hline $7 / 7$ & $10.13 \mathrm{a}$ & $6.57 \mathrm{c}$ & 8.35 & $8.67 \mathrm{a}$ & $5.43 \mathrm{~cd}$ & 7.05 \\
\hline $15 / 7$ & $7.70 \mathrm{~b}$ & $5.30 \mathrm{~d}$ & 6.50 & $6.37 \mathrm{bc}$ & $4.22 \mathrm{ef}$ & 5.30 \\
\hline $22 / 7$ & $10.20 \mathrm{a}$ & $6.63 \mathrm{c}$ & 8.42 & $8.42 \mathrm{a}$ & $5.50 \mathrm{~cd}$ & 6.96 \\
\hline Mean & 9.05 & 6.02 & & 7.60 & 4.76 & \\
\hline
\end{tabular}

Means within each column followed by the same letter(s) are not significantly different at 5\% level 


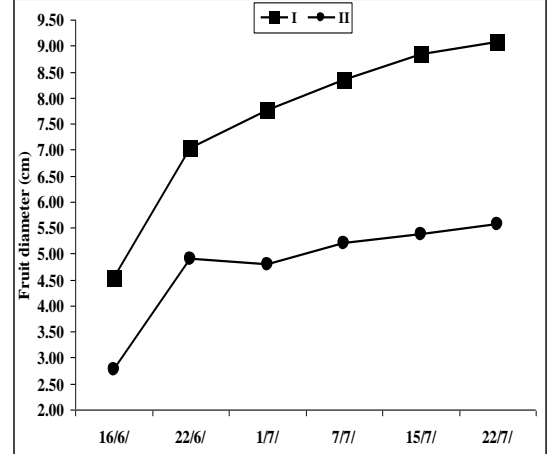

Fig. 5. Development of fruit length

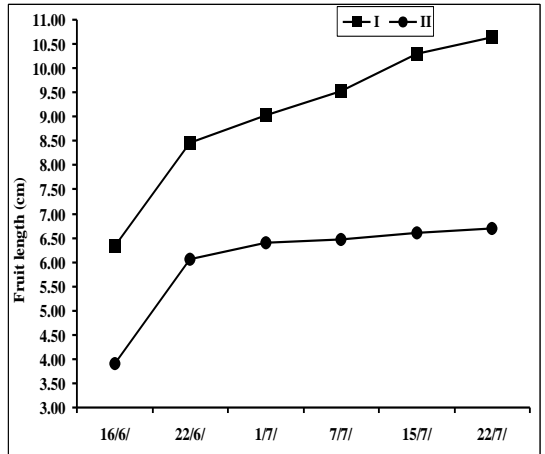

Fig. 6. Development of fruit diameter

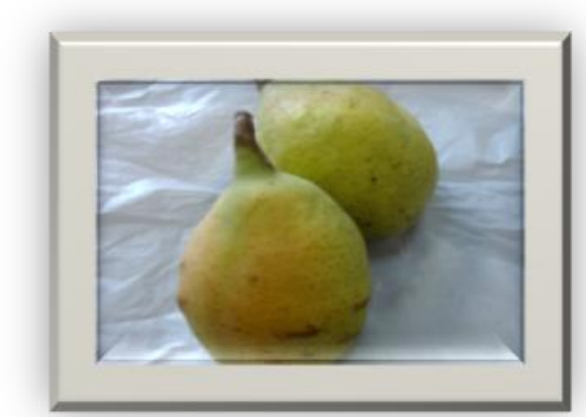

Fig.7. Fruit dimension of selected clone (22/7)

\section{Fruit chemical properties}

Fruit chemical properties of fruits is demonstrated in Table 11 Selected clone showed the highest significant TSS \% (15.67 \& 14.10) and TSS/acidity (37.32 \& 34.73 ) compared with the old cultivar in both seasons under study. While, acidity recorded a lower value in the first season and in the second season with no significant variance.

TABLE 11. Chemical properties of fruits during the two seasons $(2012 / 2013)$.

\begin{tabular}{|l|c|c|c|c|c|c|}
\hline \multirow{2}{*}{ Genotype } & \multicolumn{2}{|c|}{ T.S.S (\%) } & \multicolumn{2}{c|}{ Acidity (\%) } & \multicolumn{2}{c|}{ T.S.S/acidity } \\
\cline { 2 - 7 } & Season2012 & Season2013 & Season2012 & Season2013 & Season2012 & Season2013 \\
\hline $\begin{array}{l}\text { Selected } \\
\text { clone }\end{array}$ & $15.67 \mathrm{~A}$ & $14.10 \mathrm{~A}$ & $0.42 \mathrm{~B}$ & $0.41 \mathrm{~A}$ & $37.32 \mathrm{~A}$ & $34.73 \mathrm{~A}$ \\
\hline $\begin{array}{l}\text { Old } \\
\text { cultivar }\end{array}$ & $12.30 \mathrm{~B}$ & $12.13 \mathrm{~B}$ & $0.46 \mathrm{~A}$ & $0.43 \mathrm{~A}$ & $26.95 \mathrm{~B}$ & $28.54 \mathrm{~B}$ \\
\hline
\end{tabular}

Means within each column followed by the same letter(s) are not significantly different at $5 \%$ level

Moreover, chemical compound of fruits included total carbohydrates, total indols, total phenols ,total sugars, reducing sugars and non-reducing sugars always recorded higher values with the selected clone, when compared with the old cultivar fruits except for reducing sugars for the two seasons under study

Egypt. J. Hort. Vol. 41, No. 1 (2014) 
(Table12). Similar results were found by Fayek et al. (2004), Fayek et al. (2011) and Bahlool et al. (2000) in Le-Cont pear Cultivar.

TABLE 12. Chemical compound of fruits during seasons 2012/2013.

\begin{tabular}{|l|c|c|c|c|c|c|}
\hline Genotype & $\begin{array}{c}\text { Carbohydrates } \\
\text { /100mg }\end{array}$ & $\begin{array}{c}\text { Indols } \\
/ \mathbf{1 0 0 m g}\end{array}$ & $\begin{array}{c}\text { Phenols } \\
/ \mathbf{1 0 0 m g}\end{array}$ & $\begin{array}{c}\text { Total } \\
\text { sugars }\end{array}$ & $\begin{array}{c}\text { Reducing } \\
\text { sugars }\end{array}$ & $\begin{array}{c}\text { Non- } \\
\text { reducing } \\
\text { sugar }\end{array}$ \\
\hline $\begin{array}{l}\text { Selected } \\
\text { clone }\end{array}$ & $42.073 \mathrm{~A}$ & $0.00012 \mathrm{~A}$ & $0.07733 \mathrm{~A}$ & $5.135 \mathrm{~A}$ & $2.766 \mathrm{~B}$ & $2.369 \mathrm{~A}$ \\
\hline $\begin{array}{l}\text { Old } \\
\text { cultivar }\end{array}$ & $41.950 \mathrm{~B}$ & $0.00035 \mathrm{~B}$ & $0.0955 \mathrm{~B}$ & $4.576 \mathrm{~B}$ & $3.473 \mathrm{~A}$ & $1.103 \mathrm{~B}$ \\
\hline
\end{tabular}

Means within each column followed by the same letter(s) are not significantly different at 5\% level

Fingerprint Detected by Randomly Amplified Polymorphic DNA (RAPD)

In the present study ten primers were screened with the DNA of the new selected Clone and commercial Le-Conte pear. These primers generated reproducible and easily scorable RAPD profiles (Fig.8) .The total number of amplicon results from the ten primers was 74 with an average of 7.4 amplicon/ primer. The amplified amplicons ranged from 4 to 11 (Table 13). Polymorphic amplicons ranges from 0 to 2. Primer OPA02 and OPA17 produced the highest number of amplicons (11). While the lowest number of amplicons was produced by OPG06 (4). The highest number of polymorphic amplicons (2) produced by OPA17. While, the highest $(25 \%)$ percentage of polymorphism produced by OPG06 . The average number of polymorphic fragments was 0.3. Earlier Anna and Elzbieta (2000) identified 26 pear cultivars using RAPD markers and found that a result of reactions carried out with RAPD 25 primers, 103 polymorphic DNA fragments were obtained. The largest number of polymorphic DNA fragments (7-8) was produced in reactions with the primers OPT 15, OPG 16 and OPG 19. The size of fragments varied from 280 to1790 bp and the degree of DNA polymorphism was estimated at $56.3 \%$.

On the other hand, RAPD markers identify the selected clone and the commercial cultivar by unique positive and/or negative markers (Fig.9). Two negative unique markers were detected with the new selected clone by the OPA17. These markers were located at $550 \mathrm{bp}$ and $150 \mathrm{bp}$. Meanwhile, OPG06 revealed a unique positive marker which scored at $450 \mathrm{bp}$. Sixty decamer primers were screened, generating polymorphic patterns for 10 of 12 genotypes analyzed it was possible to find genotype-specific RAPDs and fragment patterns which could be used for cultivar identification (Oliveira et al., 1999).

To examine the genetic relationships between the two pear genotypes based on the RAPD results, the scoring data resulting from the RAPD marker were analyzed using the Dice similarity coefficient. The estimated similarities between the two genotypes were $95.3 \%$, this similarity level was high. This might be due to the use of random primers which is not accurate in the discovering of genetic differences like specific primers. 
Oliveira et al. (1999) and Khalil and Abd-Alla, 2002) realized that, cophenetic matrix computed from the tree matrix showed a significant correlation of $96.8 \%$ with the original similarity matrix. The similarities established among the different genotypes of $P$. communis and between different species were close to those usually accepted.

TABLE 13. Total number of amplicons, monomrphic amplicons, polymorphic amplicons and percentage of polymorphism revealed by RAPD markers.

\begin{tabular}{|l|c|c|c|c|c|}
\hline Primer & Sequence & $\begin{array}{c}\text { Total } \\
\text { number of } \\
\text { amplicons }\end{array}$ & $\begin{array}{c}\text { Monomorphic } \\
\text { amplicons }\end{array}$ & $\begin{array}{c}\text { Polymorphic } \\
\text { amlicons }\end{array}$ & $\begin{array}{c}\text { Percentage } \\
\text { polymorphism }\end{array}$ \\
\hline OPA-01 & TGCCGAGCTG & 7 & 7 & --- & 0.00 \\
\hline OPA-02 & AGGTGACCGT & 11 & 11 & --- & 0.00 \\
\hline OPA-03 & GTTTCGCTCC & 7 & 7 & --- & 0.00 \\
\hline OPA-06 & GGTGACGCAG & 6 & 6 & --- & 0.00 \\
\hline OPA-13 & TGGGGGACTC & 9 & 9 & --- & 0.00 \\
\hline OPA-16 & GTAGACCCGT & 5 & 5 & --- & 0.00 \\
\hline OPA-17 & GTGAGGCGTC & 11 & 9 & 2 & 18.18 \\
\hline OPA-20 & CCGCATCTAC & 7 & 7 & --- & 0.00 \\
\hline OPG-06 & TGTCATCCCC & 4 & 3 & 1 & 25.00 \\
\hline OPG-12 & GTTGCCAGCC & 7 & 7 & --- & 0.00 \\
\hline Total & & 74 & 71 & 3 & 43.8 \\
\hline Mean & & 7.4 & 7.1 & 0.3 & 4.38 \\
\hline
\end{tabular}

Similarity

\begin{tabular}{|c|c|c|}
\hline & 1 & 2 \\
\hline 1 & 100 & 95.3 \\
\hline 2 & 95.3 & 100 \\
\hline
\end{tabular}

\section{Conclusions}

The comparison among the selected clone and old cultivar showed significant differences. The selected clone described with earlier in flowering, fruit set, harvest and the highest leaf area. Also, it gave the biggest fruit weight and size, the equatorial fruit dimensions are tallest. Their chemical properties including TSS, acidity, total sugars, indols and phenols were best than in the old cultivar. It could be recommended by using of horticulture practice for increasing its yield and propagate it vegetative and studying its behavior on both Pyrus communis and Pyrus betulaefoli rootstock. The new selected clone recorded a genetic similarity with the old cultivar estimated by 95.3 .

Acknowledgment: We are deeply grateful to prof. Dr. Abd El-Rahman A. ElSheikh in Deciduous Fruit Department, Horticulture Research Institute for his supervision and kind help. 


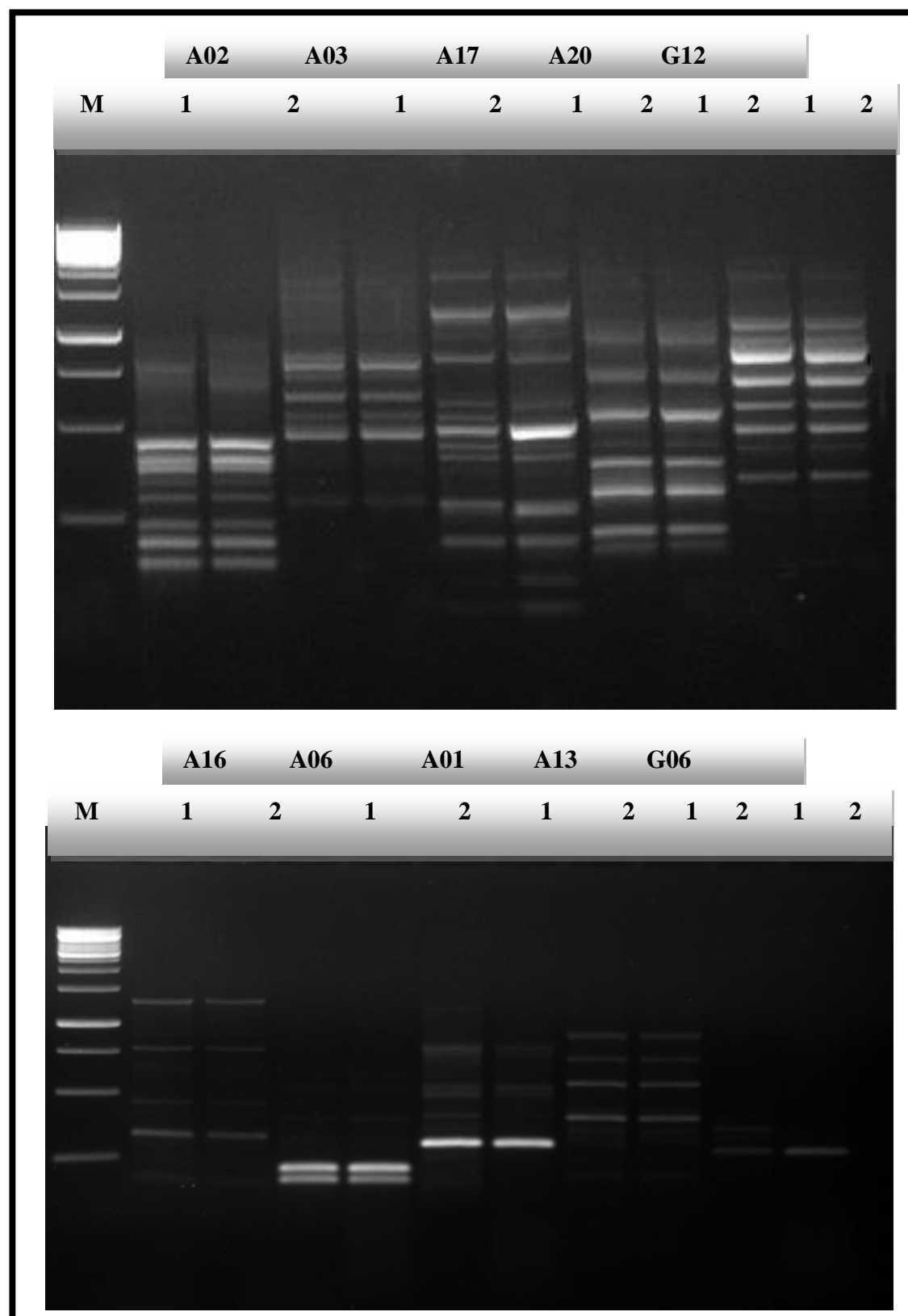

Fig. 9. RAPD profiles for the new clone and local Le-Conte cultivar as detected with primers $\mathrm{M}$ : $1 \mathrm{~Kb}$ ladder DNA

Egypt. J. Hort. Vol. 41, No. 1 (2014) 


\section{References}

Anna, L. and Elzbieta, R. (2000) Identification of pear cultivars using RAPD and ISSR markers. Journal of Fruit and Ornamental Plant Research, 18 (2), 17-22.

A.O.A.C. (2005) Official Methods of Analysis, Published by the A.O.A.C. Box 540,

Bahlool, S., Bothina A. Shahin and Kabeel, H. (2000) Effect of some cultural practices on flower bud formation, fruit set and yield of "Le- Conte" pear trees. J. Agric.Sci. Mansoura Univ., 25 (2), 975-985.

Bailey, L.H. (1923) "Various cultigens, and transfers in nomenclature". Gentes Herbarum, 1 , 113-136.

Chan-Chung, H.C., Huang-Chaur, C.W., Chen-Huang, C.C., Chio, R.W., Huang, C.C., Chen-Young, W. and Chang-lin, R. (1997) The effect of shoot bending and other cultivation practices on the lateral floral and formation of "Hasui"pear grown in Taiwan high land area. Proceeding of Symposium on enhancing competitiveness of fruit industry, Chang, Taiwan, 20-21 March1997. Special Publication-TiachungDistrect Agricultural Improvement Station, 38,187- 196.

Colaric. M., Stampar, F. and Hudina, M. (2007) Bending affects phenolic content of William pear leaves. Acta Agric.Scand., B. Soil Plant Sci., 57, 187-192.

Dubois, M.K.A., Gilles, J.K., Hamilton, P.A. and Smith, F. (1956) Colorimetric method for determination of sugars and related substances. Anal. Chem., 28, 350-354.

Evenhuis, B. (1978) Simplified Methods for Foliar Analysis" Koninklijk Institaut voorde tropen, Amsterdam, pp. 1-17

Evenhuis, B. and Dewaard, P.W. (1980) Principles and Practices in Plant Analysis Inst., Amsterdam Paper, 15, 52 -163.

Fayek, M.A., El-Shenawy, I.E. and Fayed, T.A. (2004) Inducing of flowering spurs in newly introduced Japanese pear cultivars compared to Le-Conte cultivar. Egypt. J. Applied Sci.,19, 717-729.

Fayek, M.A., Yehia, T.A., El-Fakhrany, E.M.M. and Farag, A.M. (2011) Effect of ringing and amino acids application on improving fruiting of Le-Conte pear trees. $J$. Horti. Sci. Ornamental Plants, 3,1-10

Ito, A., Yoshioka. H., Hayama, H. and Kashimura, Y. (2004) Reorientation of shoots to the horizontal position influences the sugar metabolism of lateral buds and shoot internodes in Japanese pear(Pyrus Pyrifolia (Burm.) Nak.) J. Hort. sci. Biotech., 79, 416-422

Jackson, N.L. (1967) Soil Chemical Analysis, Prentice-Hall Inc. Englewood Cliffs, NS.

Jackson, N.L. and Ulrich, A. (1959) Analytical methods for use plant analysis. Coll. of Agric., Exp. State Bull., 766, 35.

Egypt. J. Hort. Vol. 41, No. 1 (2014) 
Khalil, B.M. and Abd Alla, S.M.M. (2002) Horticultural studies and genetic fingerprinting of apricot cultivars using Random Amplified polymorohic DNA (RAPD) markers. G. Agric. Sci. Mansoura Univ., 27, 2313-2325.

Lee, S.H. (1948) A taxonomic survey of the oriental pears. Proc. Am. soc. Hort. Sci., 51, $152-155$

Li-Tain, H., Huang-Wei, D., Meng-Znac, Q., Li-Th, W.D. and Mang, Z. (1996) Study on the mechanism of flower bud induction in apple tree. Acta-PhytophysiologicaSininca, 22 (33), 251-257.

Malik, C.P. and Singh, M.B. (1980) Plant Enzymeology and Isoenzymology. A. text Manual Kaly and Publishers, New Delhi. India.

Ministry of Agriculture Statistics (2010) Agriculture Directorates of Governorates. Publisher: Economic Affairs Sector .

Mohamed, M.M., Bahan M.K. and Atef, M.M. (2012) Basateen MKM"a new early pear cultivar. Journal of American Science, 8, (12)

Murphy, S. and Riley, J.P. (1962) A modified single solution method for the determination of phosphate in natural water. Anal. Chim. Acta, 27, 31-36.

Oliveira, C.M., Motaa, M., Monte-Corvoa, L., Goula oa L. and Silvab, D.M. (1999) Molecular typing of Pyrus based on RAPD markers. Scientia Horticulturae, 79, 163174.

Pierre, E. (2001) Genotype of apple trees affects growth and fruiting responses to shoot bending at various time of year. J. Amer Soc. Hort. Sci., 125 (2), 169-174.

Sanaa, S.A. Kabeil, Mohamed, H. El-Sheikh, Elsabagh, A. Said and aboulenein, A.M. (2012) Performance of "Le Conte" Pear Trees in Response to Biological control of Fire Blight Disease . Journal of Applied Sciences Research, 8 (8), 4511-4524

Slinkard and Singleton, (1977) Total Phenol Analysis: Automation and Comparison with Manual Methods. Am. J. Enol. Vitic., 28, 149-55

Snedecor, G.W. and Cochran, G.W. (1990) Statistical Methods. $7^{\text {th }}$ ed., The Iowa state Univ. Press, Ames, Iowa, USA. 593p.

Spencer, R.D., Cross, R.J., Lumley, P.F. (2007) Plant Names, A Guide To Botanical Nomenclature. $3^{\text {rd }}$ ed., CSIRO Publishing, Collingwood. ISBN 0-643-09440-7.

Trehane, P. (2004) "50 years of the International Code of Nomenclature for Cultivated Plants". Acta Horticulturae, 634, 17-27.

Williams, J.G.K., Kublick, A.R., Livak, K.J., Rafalski, J.A. and Tingey, S.V. (1990) DNA polymorphisms amplified by arbitrary primers are useful as genetic markers. Nucleic Acids Research, 18, 6531-6535. 
Williams, J.G.K., Hanafey, M.K., Rafalski, J.A. and Tingey, S.V. (1993) Genetic analysis using random amplified polymorphic DNA markers. Methods in Enzymology, 218, 704-740.

Vanneste, J.L., Cornish D.A., Yu J. and Voyle M.D. (2002) P10c: a new biological control agent for control of fire blight which can be sprayed or distributed using honey bees. Acta Horticulturae, 590, 231-236.

Yadava, Y.L. (1986) A rapid and non -destructive method to determine chlorophyll in intact leaves. Hort. Science, 21, 1449-1450.

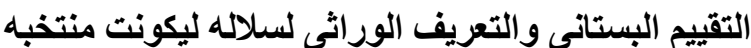

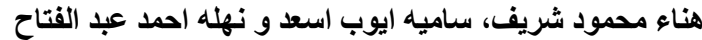

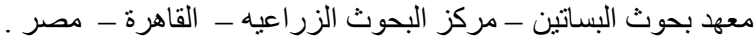

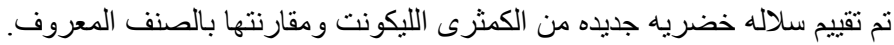

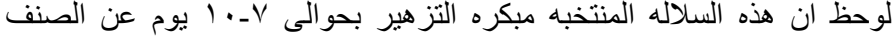

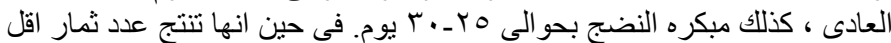

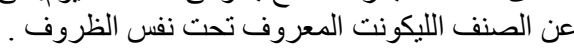

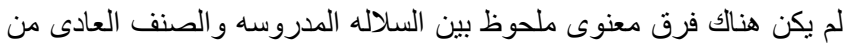

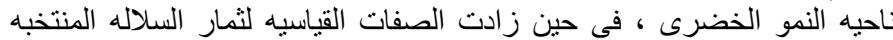

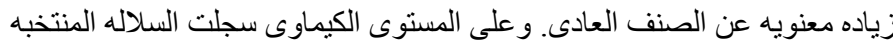

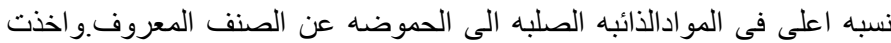
السكريا ت الكليه و المختزله نفس الاتجاه،كان المحصول الفيه القل في السلاله المنتخبه

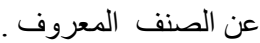

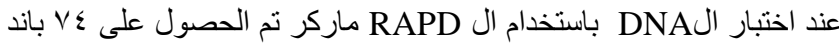

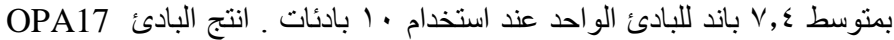

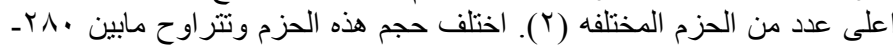
IV9.

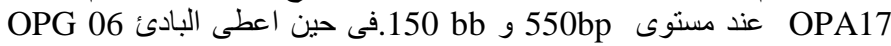

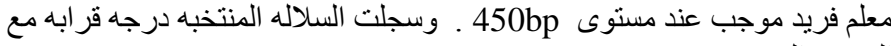

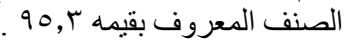

